\title{
Classificação e agrupamento das cidades brasileiras em graus-dia de aquecimento e resfriamento: 1960 a 2013
}

\author{
Classification and grouping of Brazilian cities in heating \\ and cooling degrees-day: 1960 to 2013
}

Iraci Pereira Stensjö, Camila Carvalho Ferreira, Rejane Magiag Loura

Universidade Federal de Minas Gerais, Belo Horizonte, MG, Brasil

\section{Resumo}

Graus-dia são usualmente utilizados no mundo para estimar o consumo de energia devido ao aquecimento e/ou resfriamento em edificações. Contudo, no Brasil, não há norma técnica que estabeleça um método de cálculo e/ou um zoneamento de graus-dia. Este trabalho apresenta uma proposta de cálculo do número de graus-dia aplicável em todo o país e um zoneamento nacional decorrente dessa classificação. A base de dados é composta pelas Normais Climatológicas e médias mensais do Banco de Dados Meteorológicos para Ensino e Pesquisa do INMET. Esses dados juntos permitem avaliar a evolução da condição climática das cidades. Os graus-dia de aquecimento (GDA) e resfriamento (GDR) foram calculados e as temperaturas de base foram definidas com base em um modelo adaptativo. Para a realização do agrupamento das cidades por GDA e GDR, foi utilizado o procedimento estatístico de cluster e, em seguida, a significância estatística dos agrupamentos gerados foi verificada através do Teste F. Como resultados, obteve-se a classificação das 317 cidades por número de GDA e GDR e o zoneamento proposto mostrou-se significante. Por fim, foram produzidos mapas do zoneamento das cidades brasileiras por demanda de aquecimento ou resfriamento.

Palavras-chave: Zoneamento climático. Eficiência energética. Graus-dia. Índices adaptativos.

\begin{abstract}
Degree-days is commonly used worldwide to estimate buildings energy consumption due to heating and / or cooling. However, in Brazil, there is no a technical standard calculation method and / or a degree-days zoning. The database is composed by the Climatological Norms and monthly averages of the Meteorological Database for Teaching and Research of INMET. These data allow to evaluate the evolution of the climatic condition of

IPS é Professora e coordenadora do Laboratório de Conforto Ambiental, PhD, e-mail: iraci.pereira@gmail.com CCF é Professora, Doutora, e-mail: camilaccferreira@yahoo.com.br RML é Professora, Doutora, e-mail: rejaneml@gmail.com
\end{abstract}


the cities. This paper presents a proposal for calculating degree-days applicable throughout the country, and a national zoning resulting from this classification. The heating (GDA) and cooling degree-days (GDR) were calculated based on the methodology described by Day (2006) and the temperatures were defined based on the adaptive model proposed by De Dear and Brager (2002). The grouping of cities by GDA and GDR was carried out by the cluster statistical procedure named K-means (Bussab et al., 1990) and then the statistical significance of the clusters generated was verified by the Test F. As a result, was obtained a ranking of 291 cities by number of GDA and GDR and the grouping of these seven clusters. The F test performed was significant for the proposed zoning. Finally, the zoning maps were produced in Brazilian cities by demand for heating or cooling.

Keywords: Climatic Zoning. Energy efficiency. Degrees-day. Adaptive indices.

\section{Introdução}

O setor residencial é responsável por aproximadamente $25 \%$ de toda a energia elétrica consumida pelo país. De acordo com a evolução dos dados dos balanços energéticos nacionais, o consumo de energia elétrica no setor residencial no Brasil está apresentando forte crescimento per capita, saltou de 0,455 em 2006 para 0,525 MWh/hab/ano (EPE, 2016). Por razões demográficas esse valor tende a continuar crescendo até 2030.

Outro fator que poderá impulsionar o consumo de energia elétrica do setor é o aumento do número de equipamentos de condicionamento de ar nas residências brasileiras. De acordo com a ASBRAV (2014) o Brasil apresentado crescimento significativo em vendas de equipamentos de condicionamento de ar para residências entre 2007 e 2014. É interessante exemplificar quantitativamente o impacto desta decisão de compra sobre o consumo energético de uma habitação média. 0 uso de um equipamento de ar condicionado de 7.000 BTU, durante 64 horas no mês (considerando o uso duas vezes por semana, oito horas por dia, conforme indica o Relatório de Posse do PROCEL (2007) para domicílios em climas quentes), corresponde a um consumo mensal de 57,6 kWh. Considerando o consumo da família de classe média em torno de $150 \mathrm{kWh}$ por mês, esse acréscimo significa uma elevação de $38 \%$ do consumo.

A partir deste exemplo pode-se justificar o desenvolvimento de um zoneamento climático do país baseado na demanda de condicionamento térmico das edificações, seja para resfriamento ou aquecimento. Este artigo tem por objetivo apresentar uma proposta de zoneamento climático baseada no método de graus-dia, utilizando os dados disponíveis nas Normais Climatológicas brasileiras (INMET, 2009) e no Banco de Dados Meteorológicos para Ensino e Pesquisa do INMET (INMET, 2016). Dessa forma, é possível classificar em uma base de dados mais consolidada e ampla e, posteriormente, avaliar os resultados utilizando dados mais atuais, mesmo que para um número menor de cidades.

Para tratar de maneira simplificada o impacto das temperaturas externas sobre o consumo energético das edificações, especialmente devido ao condicionamento ambiental, foram adotados os métodos de graus-hora e graus-dia. Em ambos são estabelecidas medidas quantitativas (em graus), horárias para o primeiro caso e diária para o segundo caso. Essas medidas são obtidas separadamente para análise de demanda de aquecimento e resfriamento.

Existem diferentes procedimentos de cálculo para se chegar aos valores de graus-dia. De modo geral, para tratar o impacto das baixas temperaturas, deve-se calcular graus-dias de necessidade de aquecimento (GDA). Para isso, deve-se verificar o número de dias em que a temperatura externa do ar é inferior a temperatura estabelecida como base. Já o graus-dias de necessidade de resfriamento (GDR) é calculado a partir do número de dias que a temperatura do ar exterior supera a temperatura base. É importante salientar que as temperaturas base nos cálculos de GDA e GDR são distintas. Os métodos de definição dessas temperaturas base variam em função da condição climática do local analisado.

É relevante observar que o uso de graus-dia como parâmetro para estimar o consumo tornou-se usual em diferentes países, contudo no Brasil isso ainda não ocorre. Dentre eles, a abordagem estadunidense de classificação climática baseada no cálculo de graus-dia é a mais amplamente difundida. Tanto o International Energy Conservation Code (IECC), a American Society of Heating, Refrigerating 
and Air-Conditioning Engineers (ASHRAE) e o Departamento de Energia (DOE) trabalham com um zoneamento que parte de uma classificação climática e divide o país em zonas definidas em função do número de graus-dia para aquecimento e da condição de umidade do ar. 0 primeiro zoneamento, desenvolvido pelo IECC em 2004, foi publicado na edição de 2004 da norma ASHRAE 90.1, na qual foram apresentadas oito zonas climáticas baseadas na temperatura. Além de três zonas divididas em função da condição de umidade, o que resultou em 24 divisões climáticas. Com o intuito de propor uma simplificação desta primeira versão, os pesquisadores do DOE combinaram as categorias do primeiro zoneamento e chegaram a uma classificação climática com cinco categorias. Cada uma das zonas climáticas foi definida com base no número de GDA para aquecimento, temperatura média e precipitação (DOE, 2010).

Outro tipo de utilização da metodologia de graus-dia em escala nacional ou regional visa tratar das questões relacionadas às mudanças climáticas. Há exemplos de estudos em diferentes regiões do mundo, entre elas Europa, Ásia e Austrália. No trabalho de Christenson et al. (2006) investigou-se a demanda de energia em localidades representativas daquele país a partir de graus-dia para aquecimento (GDA) e resfriamento (GDR) usando a temperatura média mensal no período de 1901 a 2003. Os resultados balizaram a criação de cenários regionais de mudanças climáticas. Pode-se constatar que de modo geral estava ocorrendo superestimação de aquecimento e subestimação de resfriamento. Observações semelhantes foram feitas por Pilli-Sihvola et al. (2010) em outros cinco países europeus: Espanha, França, Holanda, Alemanha e Finlândia. Nestes cinco contextos o trabalho demonstrou necessidade de mais energia para resfriamento e a redução de demanda para aquecimento. Em trabalho semelhante, Cartalis et al. (2001) chegou a previsões semelhantes para a Grécia.

Trabalhos realizados na Ásia também mostraram essa tendência. Lee \& Levermore (2010) analisaram duas cidades na Coreia do Sul (Seoul e Ulsan) e os resultados obtidos mostraram um significativo aumento de GDRs e consequentemente a elevação do consumo de energia e das emissões de $\mathrm{CO} 2$ devido à ampliação do uso de equipamentos de ar condicionado. Em nove zonas climáticas chinesas estudadas por Lam et al. (2011) constatou-se também a elevação de GDRs e a redução de GDAs no século 21. No ano seguinte, 2012, Lam et al (2012) em um novo trabalho realizou uma revisão das projeções e demonstrou, seguindo um método também baseado em GDA e GDR, que o maior impacto no uso de energia em edificações irá acontecer em regiões com verões quentes e invernos amenos, exatamente o caso brasileiro. 0 trabalho de Borah et al (2015) desenvolvido na Índia possibilitou o que o cálculo de graus-dias pudesse ser definido por localidade a partir de uma equação para uma zona climática, o que tem potencial para auxiliar o planejamento energético do país. Por fim, os estudos de Roshan et al (2017) apresenta novas temperaturas limites para o cálculo de graus-dia em 12 cidades representativas do país com base no diagrama de Olgyay com base em dados de 1950 e 2010.

\section{Preparação da base de dados}

O desenvolvimento de qualquer classificação climática para o Brasil enfrenta o desafio da obtenção de dados. Assim, optou-se por utilizar os dados de temperatura do ar das Normais Climatológicas 1961-1990 (INMET, 2009), pois esta possui um número importante para o contexto nacional de municípios distribuídos pelo território nacional: 291 cidades. Vale enfatizar que esse número de estações é ainda pouco representativo diante da vastidão do território nacional.

Para melhorar o comportamento estatístico dos agrupamentos, ampliamos a base de dados utilizados no cálculo de GDR e GDA. Foram acrescentadas 26 cidades existentes na base de dados das Normais Climatológicas original (DNMET, 1992), mas com lacunas de dados de temperatura na versão revista (INMET, 2009). As lacunas foram preenchidas com os dados da versão de 1992 e todo o procedimento descrito para cálculo de GDA e GDR foi realizado, o que permitiu obter então um total de 317 cidades. É importante destacar que essas cidades só foram utilizadas para melhorar o agrupamento e que a diferença entre os dados publicados entre 2009 e 1996 é muito pequena, menor que $0,2^{\circ} \mathrm{C}$ em média, valor este dentro da margem de erro de medição dos equipamentos meteorológicos.

Como os dados das normais foram publicados há quase 25 anos, optou-se por avaliar também dados mais atuais. O INMET, em seu Banco de Dados Meteorológicos para Ensino e Pesquisa permite o 
acesso a séries históricas das várias estações meteorológicas convencionais de sua rede de estações, desde 1961. Para essa pesquisa, inicialmente foi levantado uma série de 30 anos, contados a partir do momento atual. Contudo, observou-se lacunas de dados em um número elevado de estações, comprometendo a representatividade dos resultados. Assim, optou-se por utilizar uma série de 20 anos, 1993-2013, com dados para 204 cidades. Deve-se observar que essas cidades não são as mesmas da série anterior, há estações novas, e algumas estações antigas não são avaliadas, principalmente nas regiões Sul e Sudeste, em razão da lacuna de dados.

As variáveis ambientais utilizadas nesse artigo são: temperatura média compensada $\left({ }^{\circ} \mathrm{C}\right)$, temperatura máxima $\left({ }^{\circ} \mathrm{C}\right)$, temperatura mínima $\left({ }^{\circ} \mathrm{C}\right)$ e precipitação acumulada mensal (mm). Contudo, para definição dos graus-dia de aquecimento são necessários valores horários da temperatura do ar que foram obtidos a partir das temperaturas do ar máxima e mínima mensais, que levaram aos dados horários médios mensais de temperatura do ar. A partir desse valor faz-se o ajuste dos dados locais à curva-padrão da WMO, mostrada pela Eq. 1 (Assis, 2001).

$$
T_{e, j}=T_{x}-\left(T_{x}-T_{n}\right)_{K j}
$$

Onde:

$T_{e, j}$ é a temperatura do ar externa na hora $j, \mathrm{em}^{\circ} \mathrm{C}$;

$T_{x}$ é a temperatura média mensal das máximas, em ${ }^{\circ} \mathrm{C}$;

$T_{n}$ é a temperatura média mensal das mínimas, em ${ }^{\circ} \mathrm{C}$;

$k_{j}$ é o valor de ajuste da curva horária de temperatura, para a hora $\mathrm{j}$. Os valores de $\mathrm{k}$, a partir da hora 0 até a hora 23 , são: 0,$75 ; 0,78 ; 0,82 ; 0,87$; 0,$89 ; 0,96 ; 1,00 ; 0,93 ; 0,76 ; 0,57 ; 0,41 ; 0,28$; 0,$15 ; 0,11 ; 0,02 ; 0,00 ; 0,04 ; 0,08 ; 0,15 ; 0,46$; 0,$56 ; 0,64 ; 0,73 ; 0,70$.

\section{A classificação de Köppen-Greiger para o Brasil}

Inicialmente, fez-se a classificação das 317 cidades brasileiras das Normais Climatológicas original (DNMET, 1992) e das 204 cidades do Banco de Dados Meteorológicos para Ensino e Pesquisa no período de 1993-2013 de acordo com Köppen-Greiger com o intuito de permitir a comparação do agrupamento das cidades por graus-dia com um método amplamente conhecido e adotado para regionalização de variáveis climáticas. 0 processo utilizado baseou-se na metodologia descrita por Kottek et al. (2006) e Peel at al. (2007) e as chaves para sua reprodução são expostas na Tabela 1. Os mapas com as classificações climáticas por Köppen-Greiger são mostrados na Figura 1.

Tabela 1 - Chaves para a classificação do clima conforme Köppen e Greiger

\begin{tabular}{|c|c|c|c|c|}
\hline & Tipo & & Descrição & Critério \\
\hline \multirow[t]{5}{*}{ A } & & & Tropical & $\operatorname{Tmin} \geq 18^{\circ} \mathrm{C}$ \\
\hline & $f$ & & equatorial & $P \min \geq 60 \mathrm{~mm}$ \\
\hline & m & & monção & Não (Af) e Pano $\geq 25$ (100-Pmin) \\
\hline & $\mathbf{w}$ & & $\begin{array}{l}\text { Savana (estação seca de } \\
\text { inverno) }\end{array}$ & Pimin,$<60 \mathrm{~mm}$ \\
\hline & $\mathbf{s}$ & & $\begin{array}{l}\text { Savana (estação seca } \\
\text { de verão) }\end{array}$ & Pvmin $<60 \mathrm{~mm}$ \\
\hline \multirow[t]{5}{*}{ B } & & & Climas áridos & Pano < 10Plim \\
\hline & S & & Estepes & Pano $>$ 5Plim \\
\hline & W & & Desertos & Pano $\leq 5$ Plim \\
\hline & & h & $\begin{array}{l}\text { quentes de baixa latitude } \\
\text { ou altitude }\end{array}$ & $\mathrm{TMA} \geq 18^{\circ} \mathrm{C}$ \\
\hline & & k & $\begin{array}{l}\text { frio das latitudes médias } \\
\text { ou de grande altitude }\end{array}$ & $\mathrm{TMA}<18^{\circ} \mathrm{C}$ \\
\hline \multirow[t]{9}{*}{ C } & & & Temperado & $\operatorname{Tmax}>10$ e $0<T \min <18 \mathrm{C}$ \\
\hline & & & & Pvmin $<$ Pimin, Pimax $>3$ Pvmin e \\
\hline & s & & veluo seco & Pvmin $<40 \mathrm{~mm}$ \\
\hline & & & & Pimin $<$ Pvmin and Pvmax $>10$ \\
\hline & w & & Inverno seco & Pimin \\
\hline & $f$ & & Sem estação seca & Não (Cs ou CW) \\
\hline & & a & Verão quente & $\operatorname{Tmax} \geq 22^{\circ} \mathrm{C}$ \\
\hline & & b & Verão ameno & Não (a) e Tmes $10 \geq 4$ \\
\hline & & c & Verão frio & Não ( $a$ e b) e $1 \leq$ Tmes $10<4$ \\
\hline
\end{tabular}

Convenções: Tmin: menor temperatura média no ano; Tmax: maior temperatura média no ano; TMA: temperatura média anual; Pmin: menor precipitação mensal no ano; Tmes10: número de meses com temperatura superior à $10^{\circ} \mathrm{C}$; Pano: precipitação total anual; Plim: Precipitação limite, (varia conforme as regras: se $70 \%$ da Pano ocorre no inverno, Plim = 2TMA; se 70\% da Pano ocorre no verão, Plim= 2TMA + 28; de outra forma, Plim= $2 T M A+14)$; Pimin: menor precipitação mensal nos meses de inverno; Pvmin: menor precipitação mensal nos meses de verão; Verão (ou inverno) é definido como o semestre mais quente (frio) de ONDJFM e AMJJAS.

Fonte: Adaptado de Kottek et al. (2006) e Peel at al. (2007). 


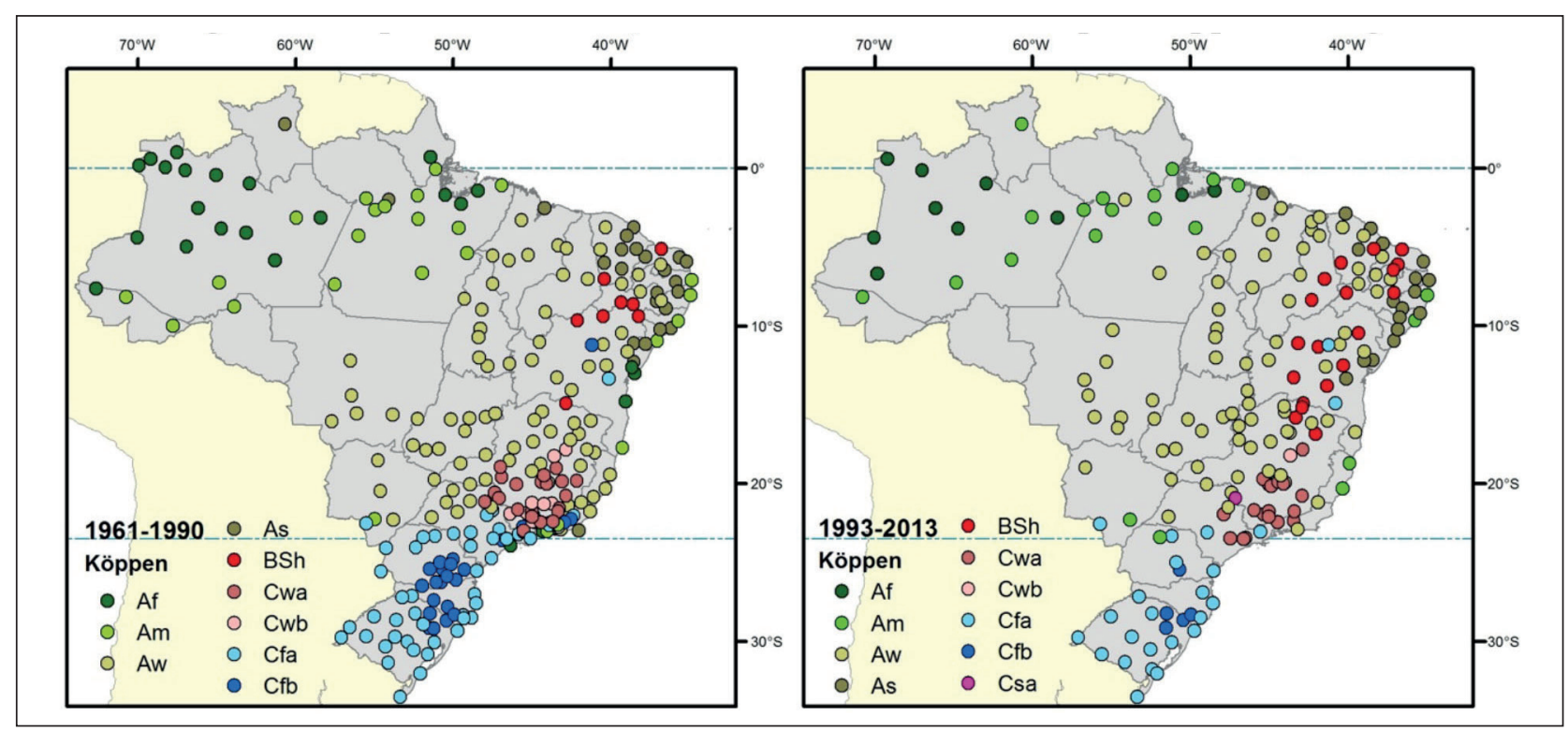

Figura 1- Mapa da classificação climática das estudadas por Köppen- Greiger. Fonte: Elaboraç̦̃óo própria.

Pelos mapas, percebe-se que ao norte do país predominam os climas mais quentes e úmidos (Af e Am), ao sul, os climas temperados sem estação seca (Cfa e Cfb) e ao centro do país, clima de savana (Aw). Os climas temperados com estação seca (Cwa e Cwb) localizam-se na Região Sudeste, principalmente em Minas Gerais. Na Região Nordeste, há uma variação de climas quentes, com predomínio do Equatorial no litoral, passando pelos tropicais de savana (Aw e As), até atingir o clima de estepes (BSh) na sua área central.

As principais variações entre os dois mapas ocorreram, em geral, na Região Sudeste, com destaque para as estações em Minas Gerais. Há redução de ocorrências de climas temperados e aumento de climas quentes (Aw) e de estepes (BSh) a norte. Também ocorre o surgimento de um temperado úmido com verão quente e seco (Csa) a sul. No litoral do Espirito Santo, alguns locais que anteriormente eram climas de savanas aparecem como clima quente e úmido (Am). É importante destacar que há menos localidades na segunda classificação, isso explica, entre outros, a redução significativa de cidades em Cfa e Cfb na Região Sul.

\section{Cálculo do número de horas de GDA e GDR}

De acordo com Day (2006), o método mais rigoroso (e mais preciso matematicamente) de se calcular graus-dia é somar as diferenças horárias entre as temperaturas do ar e a temperatura de base e dividir por 24. Nesse cálculo, é importante que apenas os valores positivos sejam somados. Para calcular o número de graus-dia de aquecimento (GDA) quando a temperatura externa for superior à temperatura de base, o valor adotado de GDA para esta hora é zero. A Eq. 2 mostra a fórmula geral para este processo, considerando GDA.

$$
D_{d}=\frac{\sum_{j=1}^{24}\left(T_{b}-T_{e, j}\right)_{\left[\left(T_{b}-T_{e, j}\right)>0\right]}}{24}
$$

Onde:

$D_{d}$ é o número de graus-dia diário para um dado dia;

$T_{b}$ é a temperatura de base;

$T_{e, j}$ é a temperatura do ar externa na hora $j$;

A parte subscrita denota que apenas valores positivos serão considerados. 
Para graus-dia de resfriamento (GDR), a Eq. 2 se torna a Eq. 3.

$$
D_{d}=\frac{\sum_{j=1}^{24}\left(T_{e, j}-T_{b}\right)_{\left[\left(T_{e, j}-T_{b}\right)>0\right]}}{24}
$$

Os graus-dia são então somados pelo período definido, usualmente por um mês, uma estação ou um ano. Neste artigo, o número de graus-dia foi calculado para cada dia normal, definição já apresentada. Esse valor foi multiplicado pelo número de dias do mês e a soma efetuada para todos os meses do ano, para se obterem os valores anuais de GDR e GDA, conforme mostrado na Eq. 4.

$$
D_{a}=\sum_{\mathrm{i}=1}^{12}\left(\mathrm{D}_{\mathrm{d}, \mathrm{i}} \mathrm{d}_{\mathrm{i}}\right)
$$

Onde:

$D_{a}$ é o número de graus-dia total no ano;

$D_{d, i}$ é o número de graus-dia calculado para o dia normal do mês $i$;

$d_{i}$ é o número de dias do mês $i$.

\section{Definição das temperaturas de base}

Por causa da grande diversidade de climas do Brasil, havendo desde locais onde há apenas demanda de resfriamento, até locais onde há grande demanda de aquecimento nas edificações. Optouse por definir as temperaturas de base para aquecimento e resfriamento a partir da temperatura de neutralidade, pois esse tipo de modelo de conforto térmico permite refletir as condições de aclimatação das populações de climas diferentes.

A temperatura de neutralidade pode ser definida por meio do modelo adaptativo de De Dear \& Brager (2002), mostrado na Eq. 5. Esse trabalho segue o modelo tradicional de temperatura neutra, no qual Tn é uma temperatura do ar de neutralidade, definido pela temperatura média mensal externa, medido por estações meteorológicas locais. Destaca-se que este é o modelo clássico de determinação da temperatura neutra, presente em diferentes trabalhos como o de Auliciems (1981),
Humphreys (1978) Nicol \& Humphreys (2002) e De Dear \& Brager (2002).

$$
T_{n}=0,31 T_{e}+17,8^{\circ} \mathrm{C}
$$

\section{Onde: \\ $T_{n}$ é a temperatura de conforto ou neutralidade; \\ $T_{e}$ é a temperatura média mensal externa.}

Conforme discutido por Pereira \& Assis (2010), a temperatura neutra apresenta boa aproximação com os limites de conforto estudados para o Brasil por diferentes autores, em cidades com diferentes condições climáticas.

De Dear \& Brager (2002) ainda definem faixas de aceitabilidade a partir da temperatura de neutralidade. As temperaturas internas devem estar dentro da faixa de $\mathrm{Tn} \pm 2,5{ }^{\circ} \mathrm{C}$, para uma aceitabilidade de $90 \%$ dos usuários. Para $80 \%$ de aceitabilidade, as temperaturas internas devem estar dentro da faixa de $\mathrm{Tn} \pm 3,5{ }^{\circ} \mathrm{C}$. Neste trabalho, a temperatura de base para o cálculo de graus-dia de resfriamento selecionada é $\mathrm{Tn}+2,5^{\circ} \mathrm{C}$. Para aquecimento, a adoção do limite de conforto de $\mathrm{Tn}-3,5{ }^{\circ} \mathrm{C}$ mostrou-se inadequada, por gerar uma temperatura base muito elevada, levando a uma demanda de aquecimento, mesmo em cidades de climas quente-úmido como Belém. Nos diferentes trabalhos existentes no mundo sobre zoneamento por graus-dia e apresentados no item 1 , a temperatura de base adotada para calcular o GDA é $18^{\circ}$ C, mas não há nestes estudos a indicação um estudo científico que justificasse tal escolha.

Considerou-se incoerente adotar um limite fixo para temperatura de base de aquecimento, se a temperatura de base para resfriamento foi definida pela temperatura de neutralidade. Por isso, estabeleceu-se o limite de $\mathrm{Tn}-5^{\circ} \mathrm{C}$ para GDA, uma vez que esse valor não gera demanda de aquecimento em locais de clima predominantemente quentes, como os climas Am e Af. Além disso, esse limite gera temperaturas de base próximas aos 18 ${ }^{\circ} \mathrm{C}$ em cidades de climas temperados brasileiros (climas Cf e Cw por Köppen e Greiger), ou seja, as cidades com climas mais semelhantes aos dos estudos levantados no item, em geral. 


\section{Agrupamentos de dados}

Tendo-se calculado o número de GDR e GDA para as cidades estudadas, faz-se necessária a classificação das cidades por similaridade de demanda de aquecimento e/ou resfriamento dentro do país.

Para melhorar o comportamento estatístico dos agrupamentos, a base de dados utilizados no cálculo de GDR e GDA foi ampliada. Foram acrescentadas 74 cidades existentes na base de dados das Normais Climatológicas original (DNMET, 1992), mas com lacunas de dados de temperatura na versão revista (INMET, 2009). As lacunas foram preenchidas com os dados da versão de 1992 e todo o procedimento descrito para cálculo de GDA e GDR foi realizado, o que permitiu obter um total de 317 cidades. É importante destacar que essas cidades só foram utilizadas para melhorar o agrupamento e que a diferença entre os dados publicados entre 2009 e 1996 é muito pequena, menor que $0,2^{\circ} \mathrm{C}$ em média, valor que está dentro da margem de erro de medição dos equipamentos meteorológicos.

Para o agrupamento adotou-se o conceito de grupo ou cluster, que pode ser entendido como um conjunto de dados em que seus elementos possuam características em comum. Assim, o agrupamento de dados busca revelar inter-relações entre amostras de um dado universo, quando se desconhece a priori o número de grupos. Na busca de padrões de dados para a identificação de agrupamentos, a Análise de Cluster é uma das técnicas mais utilizadas, sendo uma técnica de classificação de padrões dentro de grupos. Seu objetivo principal é agrupar as observações em classes naturais de forma que os elementos pertencentes a um mesmo grupo tenham alto grau de semelhança ou similaridade, enquanto que, quaisquer elementos pertencentes a grupos distintos tenham pouca semelhança entre si (Reddy, 2011).

Para se definir os clusters são utilizadas medidas de distância entre os elementos, sendo que quanto menor a distância, mais semelhantes serão os elementos e estes deverão ficar no mesmo cluster. De forma análoga, quanto maior a distância, menor a similaridade dos elementos, devendo então estar em clusters distintos. A distância usualmente adotada é a distância Euclidiana (Reddy, 2011).

No presente trabalho adotou-se o algoritmo particionado, em razão do objetivo não ser o agrupamento em níveis, pelo procedimento estatístico de cluster denominado de K-means, método simples e efetivo. 0 procedimento K-means é uma associação de médias para se identificar grupos de casos relativamente homogêneos, baseado em características selecionadas. A associação ocorre através de um algoritmo capaz de lidar com um grande número de casos, que relaciona os casos em análise com os centros geométricos dos clusters ou grupos. Assim, uma população heterogênea é particionada em vários subgrupos mais homogêneos, de acordo com a semelhança. Neste caso a medida de similaridade é a distância geométrica no espaço multidimensional das médias (Bussab et al., 1990). 0 método trabalha com o agrupamento de casos em $\mathrm{k}$ grupos, onde ké a quantidade de clusters ou grupos definidos previamente. Não há uma metodologia estabelecida para a definição do número de clusters, devendo ser este pré-estabelecido pelo pesquisador e sua validade testada posteriormente. Nem todos os valores adotados para k resultarão em um agrupamento satisfatório, havendo, então, a necessidade de aplicar o método várias vezes para diferentes valores de k, optando por aquele agrupamento que se mostrar mais representativo (Bussab et al. 1990). Define-se como representativos agrupamentos que: (a) os grupos devem abranger todas as observações; (b) o extremo superior de um grupo deve ser o extremo inferior da classe subsequente; (c) cada valor observado deve enquadra-se em apenas um grupo; e (d) a quantidade de grupos, de modo geral, não deve ser inferior a 5 ou superior a 25 (Farias et al, 2003).

A significância estatística dos agrupamentos resultantes foi feita por meio do Teste F, que relaciona a variabilidade entre os grupos com a variabilidade dentro do grupo, cujo valor $\mathrm{F}$ deve ser um número bem superior à unidade para comprovar que o agrupamento gerado possui esta característica.

\section{Resultados}

A variação do número de graus-dia obtida é apresentada nas Figuras 2 e 3, para GDA e GDR, respectivamente, para as séries de 1961-90 e 19932013. Os mapas por séries são apresentados lado a lado, e utilizam a mesma escala, para permitir melhor comparação. 


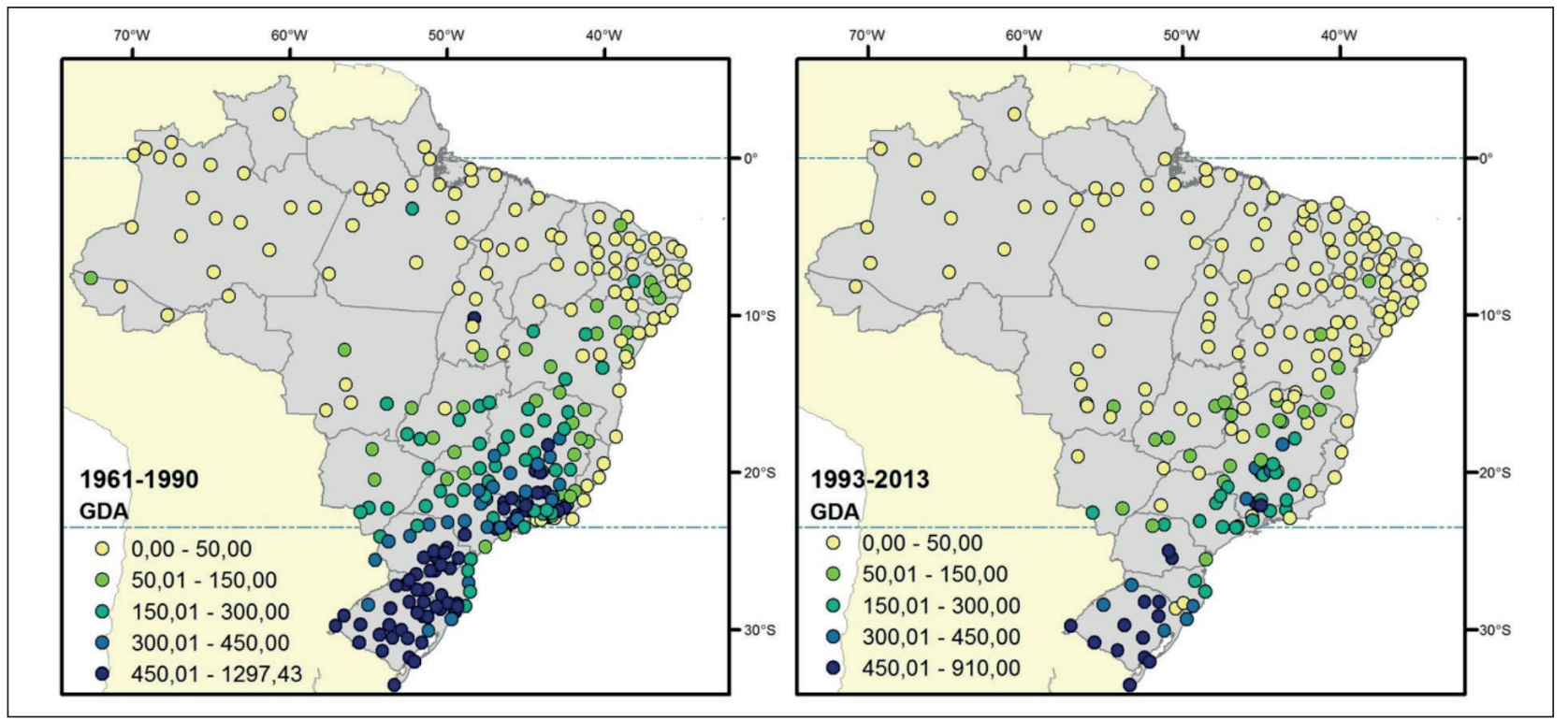

Figura 2 - Mapa com a variação da distribuição dos GDA no território nacional. Fonte: Elaboração própria.

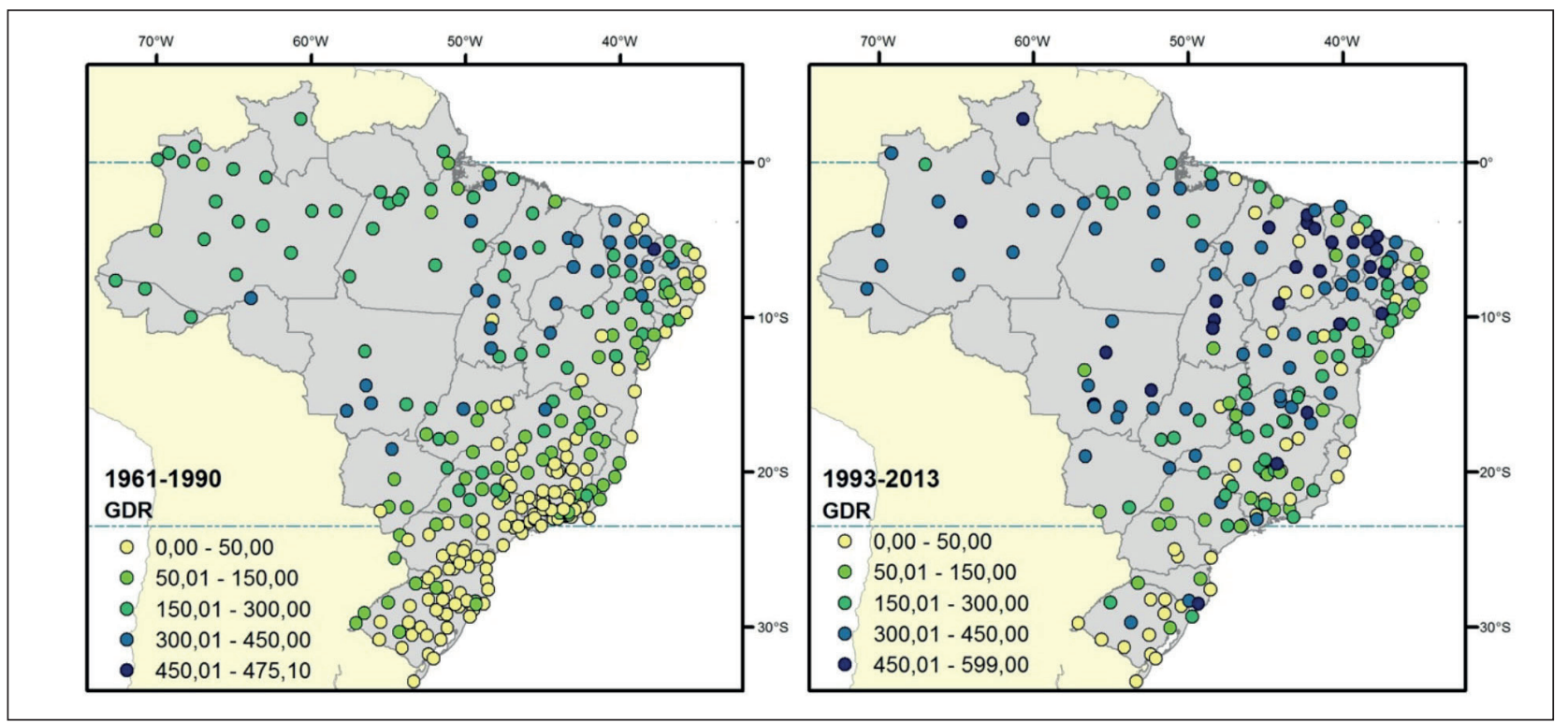

Figura 3 - Mapa com a variação da distribuição dos GDR no território nacional. Fonte: Elaboração própria.

Para o período de 1961-1990, observa-se que a Região Norte é a que possui maior demanda de resfriamento e menor demanda de aquecimento. Na Região Sul, essa relação é oposta. Na Região Sudeste, há uma grande variação nos valores de GDA e GDR entre cidades próximas, principalmente no estado de MG, fato explicado por sua variação climática e de altitude. Nas regiões Centro-Oeste e Nordeste há predomínio de elevada demanda de resfriamento.

Para o período de 1993-2013 a distribuição espacial de GDA é semelhante ao período anterior. A maior variação ocorre na Região Sudeste, onde observa-se uma redução do número de horas de 
aquecimento nas estações existentes. Para resfriamento, observa-se uma tendência geral de aumento do número de GDR nas estações estudadas.

\section{Agrupamentos}

Para a demanda de refrigeração (GDR) decidiu-se por testar agrupamentos de 3, 5 e 7 grupos e avaliar qual apresentaria maior representatividade, conforme apresentado a seguir. Os agrupamentos foram gerados no software estatístico SPSS versão 16.
O primeiro agrupamento a ser testado foi o com três grupos. Após o agrupamento gerado, avaliou-se a não existência de dados que apresentam comportamento diferenciado da média, os chamados outleirs. Para os dados de 1961-1990, nesta proposta de agrupamento, conforme a Tabela 2, a concentração de cidades no Grupo 3 e um desvio padrão alto para todos os três conjuntos, o que permite concluir que essa divisão é pouco representativa, indicando a necessidade de um número maior de grupos. Para os dados de 1993-2013, a concentração de cidades em um único grupo não foi observada, contudo o desvio padrão alto para todos os três conjuntos também foi observado para estes dados.

Tabela 2 - Distribuição dos casos no agrupamento de 3 clusters para GDR

\begin{tabular}{ccccccc}
\hline \multirow{2}{*}{ Cluster Dados de 1961-1990 } & \multicolumn{3}{c}{ Dados de 1993-2013 } \\
\cline { 2 - 7 } & $\mathbf{n}^{\mathbf{0}}$ de observações & Centro geométrico & Desvio padrão & $\mathbf{n}^{\circ}$ de observações & Centro geométrico & Desvio padrão \\
\hline 1 & 63 & 309,53 & 56,7 & 72 & 58,70 & 47,09 \\
2 & 94 & 153,10 & 41,1 & 61 & 249,96 & 57,53 \\
3 & 160 & 25,24 & 27,7 & 71 & 442,62 & 61,14 \\
\hline Total & 317 & & & & \\
\hline
\end{tabular}

Fonte: Elaboração própria.

Frente a essa condição, propôs-se a divisão em cinco grupos da população de dados, conforme Tabela 3. Pode-se perceber a ausência de concentração de dados em um único grupo e a redução do desvio padrão em cada um dos grupos para as duas bases de dados climáticos, havendo assim uma melhora no agrupamento com este número de clusters.
A diferença observada entre as bases de dados é o maior desvio padrão no período 1993-2013. Nessa distribuição também notou-se a inexistência de outleirs. Dessa forma, pode-se afirmar que esse agrupamento tem melhor representatividade que o anterior, nos dois casos.

Tabela 3 - Distribuição dos casos no agrupamento de 5 clusters para GDR

\begin{tabular}{ccccccc}
\hline \multirow{2}{*}{ Cluster } & \multicolumn{3}{c}{ Dados 1961-1990 } & \multicolumn{3}{c}{ Dados 1993-2013 } \\
\cline { 2 - 7 } & $\mathbf{n}^{\mathbf{0}}$ de observações & Centro geométrico & Desvio padrão & $\mathbf{n}^{\circ}$ de observações & Centro geométrico & Desvio padrão \\
\hline 1 & 47 & 324,66 & 28,45 & 55 & 37,45 & 30,24 \\
2 & 73 & 121,82 & 30,21 & 40 & 162,24 & 33,72 \\
3 & 13 & 228,97 & 28,40 & 38 & 287,48 & 35,37 \\
4 & 134 & 22,51 & 21,39 & 45 & 403,90 & 32,37 \\
5 & 50 & 433,49 & 39,23 & 26 & 509,63 & 35,37 \\
\hline Total & 317 & \multicolumn{5}{c}{204} \\
\hline
\end{tabular}

Fonte: Elaboração própria. 
Por fim, foi analisada a divisão em sete grupos, visando a avaliação de sua representatividade, conforme mostrado na Tabela 4, com a distribuição dos casos nesse agrupamento. Como nos outros casos, não houve a presença de outleirs. Em relação ao agrupamento de 5 clusters, para ambas as bases de dados climáticos, pode-se observar a redução do desvio padrão. Apesar disso, essa divisão originou cluster com apenas sete cidades (Cluster 7) para os dados de 1961-1990, sendo este número de observações pouco significativo para o universo trabalhado de 317 cidades. Por isso, pode-se dizer que este grupo é pouco representativo. Em razão disto, adotou-se a divisão em cinco clusters para o agrupamento das cidades a partir da necessidade de resfriamento, para ambas bases de dados.

Tabela 4 - Distribuiç,ão dos casos no agrupamento de 7 clusters para GDR

\begin{tabular}{ccccccc}
\hline \multirow{2}{*}{ Cluster } & \multicolumn{3}{c}{ Dados 1961-1990 } & \multicolumn{3}{c}{ Dados 1993-2013 } \\
\cline { 2 - 7 } & $\mathbf{n}^{\mathbf{0}}$ de observações & Centro geométrico & Desvio padrão & $\mathbf{n}^{\mathbf{0}}$ de observações & Centro geométrico & Desvio padrão \\
\hline 1 & 32 & 261,77 & 17,8 & 42 & 24,06 & 20,04 \\
2 & 46 & 127,43 & 18,0 & 30 & 107,2 & 26,28 \\
3 & 54 & 68,52 & 18,6 & 32 & 202,11 & 26,94 \\
4 & 114 & 9,66 & 11,1 & 27 & 299,73 & 26,64 \\
5 & 26 & 330,4 & 23,1 & 29 & 379,32 & 22,49 \\
6 & 38 & 192,24 & 20,2 & 27 & 448,15 & 19,37 \\
7 & 7 & 427,806 & 32,3 & 17 & 530,14 & 25,23 \\
\hline Total & 317 & \multicolumn{5}{c}{} \\
\hline
\end{tabular}

Fonte: Elaboração própria.

A divisão dos dados em sete grupos, embora tenha apresentando uma redução no desvio padrão, originou cluster com apenas sete cidades (Cluster 7) para os dados de 1961-1990, sendo este número de observações pouco significativo para o universo trabalhado de 317 cidades. Por isso, pode-se dizer que este grupo é pouco representativo. Em razão disto, adotou-se a divisão em cinco clusters para o agrupamento das cidades a partir da necessidade de resfriamento.

No caso do agrupamento das cidades de acordo com a necessidade de aquecimento, GDA, optou-se pela separação das cidades em apenas três grupos para a base de dados das Normais Climatológicas, devido ao predomínio do clima tropical no Brasil, havendo poucas cidades com significativa necessidade de aquecimento: sem necessidade de aquecimento, com baixa necessidade de aquecimento e com alta necessidade de aquecimento. Estes três grupos se fazem suficientes para abordar toda a amostra. Quando se agrupam as cidades em mais de três conjuntos, originam-se grupos pouco representativos que abrangem um pequeno número de cidades. No entanto, para a base de dados do INMET, apenas no caso do agrupamento em dois conjuntos não ocorreram grupos pouco representativos. Por isso, essa foi a divisão adota para GDA. 0 agrupamento das cidades conforme GDA está apresentado na Tabela 5.

Tabela 5 - Distribuição dos casos no agrupamento de 3 clusters para GDA

\begin{tabular}{ccccc}
\hline \multirow{2}{*}{ Cluster } & \multicolumn{2}{c}{ Dados 1961-1990 } & \multicolumn{2}{c}{ Dados 1993-2013 } \\
\cline { 2 - 5 } & $\mathbf{n}^{\circ}$ de observações & Centro geométrico & $\mathbf{n}^{\circ}$ de observações & Centro geométrico \\
\hline 1 & 201 & 62,89 & 186 & 58,97 \\
2 & 78 & 388,96 & 18 & 806,21 \\
3 & 38 & 782,26 & - & - \\
\hline Total & 317 & & 204 & \\
\hline
\end{tabular}

Fonte: Elaboração própria. 


\section{O agrupamento das cidades por GDR e GDA}

A partir dos agrupamentos obtidos separadamente, pode-se comparar a ocorrências das cidades nos respectivos grupos de GDR e GDA. Um ponto relevante observado refere-se ao fato de que as bases de dados climáticos resultaram em diferentes agrupamentos.

Para a base de dados climáticos de 1961-1990, analisando as ocorrências das cidades em cada grupo (Tabela 6), constatou-se que as cidades podiam ser divididas de acordo com a necessidade de
GDR (baixa, média e alta), de GDA (baixa e alta) e de neutralidade. Deste modo, o agrupamento final das cidades de acordo com a necessidade de refrigeração e de aquecimento resultante foi o de seis grupos, denominados e classificados conforme descrição da Tabela 7.

0 Teste $\mathrm{F}$ realizado (Tabela 8) para o agrupamento das cidades por GDR e GDA para a base de dados de 1961-1990 mostrou-se significante, uma vez que a variância dentro dos grupos é bastante inferior à variância obtida entre os grupos, resultando em um valor de $\mathrm{F}$ muito superior a 1.

Tabela 6 - Ocorrência de GDA nos respectivos grupos de GDR a partir do agrupamento gerado para os dados de 1961-1990

\begin{tabular}{ccccc}
\hline \multirow{2}{*}{ Cluster GDR } & \multicolumn{2}{c}{ Demanda de aquecimento } & \multirow{2}{*}{ TOTAL } \\
\cline { 2 - 4 } & Alto GDA & Baixo GDA & Não há GDA & \\
\hline 1 & 0 & 01 & 46 & 47 \\
2 & 0 & 19 & 54 & 73 \\
3 & 0 & 0 & 13 & 13 \\
4 & 38 & 56 & 40 & 134 \\
5 & 0 & 02 & 48 & 50 \\
\hline Total & 38 & 78 & 201 & 317 \\
\hline
\end{tabular}

Fonte: Elaboração própria.

Tabela 7 - Agrupamento para as 317 cidades conforme necessidade de aquecimento e resfriamento para os dados de 1961-1990

\begin{tabular}{cccc}
\hline Grupo & Necessidade & Valor (em horas) & Ocorrências \\
\hline GDA 1 & Demanda de aquecimento baixa & GDA $<608,7$ horas & 75 \\
GDA 2 & Demanda de aquecimento alta & GDA $>608,7$ horas & 38 \\
GDR 1 & Demanda de resfriamento baixa & GDR $<246,0$ horas & 50 \\
GDR 2 & Demanda de resfriamento média & GDR $<353,3$ horas & 47 \\
GDR 3 & Demanda de resfriamento alta & GDA $>475,7$ horas & 13 \\
Neutro & Sem demanda de aquecimento e & GDR $<148,3$ horas e GDA $<236,6$ & 91 \\
\hline
\end{tabular}

Fonte: Elaboraç̃̃o própria.

Tabela 8 - Teste de significância para 0 agrupamento gerado para os dados de 1961-1990

\begin{tabular}{ccc}
\hline $\mathbf{S}_{\text {ENTRE }}$ & $\mathbf{S}_{\text {DeNTRO }}$ & $\mathbf{F}$ \\
\hline 1680038,1 & 1346,4 & 1247,8 \\
\hline
\end{tabular}

Fonte: Elaboracão própria.
Já para a base de dados climáticos de 19932013, as ocorrências das cidades em cada grupo indicaram também a existência de seis grupos, mas divididos de acordo com a necessidade de GDR (baixa, média, alta e muito alta), a necessidade de GDA e a de neutralidade (Tabela 9). Os seis 
grupos, denominados e classificados conforme descrição são apresentados na Tabela 9.
0 Teste $\mathrm{F}$ realizado (Tabela 10 ) para o agrupamento das cidades por GDR e GDA para a base de dados de 1993-2013 mostrou-se igualmente significante.

Tabela 9 - Agrupamento para as 204 cidades conforme necessidade de aquecimento e resfriamento para os dados de 1993-2013

\begin{tabular}{cccc}
\hline Grupo & Necessidade & Valor (em horas) & Ocorrências \\
\hline GDA 1 & Demanda de aquecimento & GDA $>1596,7$ horas & 17 \\
GDR 1 & Demanda de resfriamento baixa & GDR $<224,5$ horas & 40 \\
GDR 2 & Demanda de resfriamento média & $G D R<343,5$ horas & 38 \\
GDR 3 & Demanda de resfriamento alta & GDR $<456,5$ horas & 45 \\
GDR 4 & Demanda de resfriamento muito alta & GDR $>459,8$ horas & 26 \\
Neutro & Sem demanda de aquecimento e & GDR $<94,4$ horas e GDA $<404,1$ & 91 \\
\hline
\end{tabular}

Fonte: Elaboração própria.

Tabela 10 - Teste de significância para 0 agrupamento gerado para os dados 1993-2013

\begin{tabular}{ccc}
\hline $\mathbf{S}_{\text {ENTRE }}^{2}$ & $\mathbf{S}_{\text {DeNTRO }}$ & $\mathbf{F}$ \\
\hline 1416148,4 & 1116,3 & 1268,6 \\
\hline
\end{tabular}

Fonte: Elaboração própria.

A principal diferença observada entre os agrupamentos obtidos a partir das duas bases de dados climáticos refere-se à extinção do grupo de baixa demanda de aquecimento e o surgimento de um grupo de muito alto resfriamento na base de dados de 1993-2013.

A partir da definição final dos clusters, os dados foram inseridos no software ArcView, para permitir a visualização espacial dos resultados. 0 mapa (Figura 4) mostra que as cidades com demanda de aquecimento, localizam-se, principalmente, nas Regiões Sul e Sudeste, em locais de clima temperado ( $\mathrm{Cf}$ ou $\mathrm{Cw}$ ), conforme mostra Figura 1. As cidades com menor de demanda de resfriamento (Neutro ou GDR1) estão espalhadas por todo o território nacional, em climas equatoriais, tropicais e temperados. Nessas cidades, a temperatura do ar está, predominantemente, próxima à temperatura de neutralidade térmica, por isso, a demanda para uso de sistemas de climatização artificial (aquecimento e/ou resfriamento) é nula ou pequena. As cidades com maior demanda de resfriamento (GDR 2 e 3) ocorreram principalmente nos climas tropicais de savana, como também nos climas equatoriais e de estepes.

Ao se comparar os dois mapas da Figura 4 percebe-se que ocorre demanda de aquecimento reduzida nas regiões Sul e Sudeste. A demanda na Região Norte e Nordeste por resfriamento aumenta, passando de média a muito alta. As maiores alterações ocorreram na Região Sudeste, com destaque para as estações localizadas em Minas Gerais, fato já notado no mapa com a classificação de Köppen. Nessa Região, praticamente não há mais demanda de aquecimento e cresce consideravelmente os locais classificados em demanda de resfriamento moderada. Os valores do segundo mapa são mais recentes e podem ser, em alguma medida, indícios de alterações climáticas nas cidades brasileiras. 


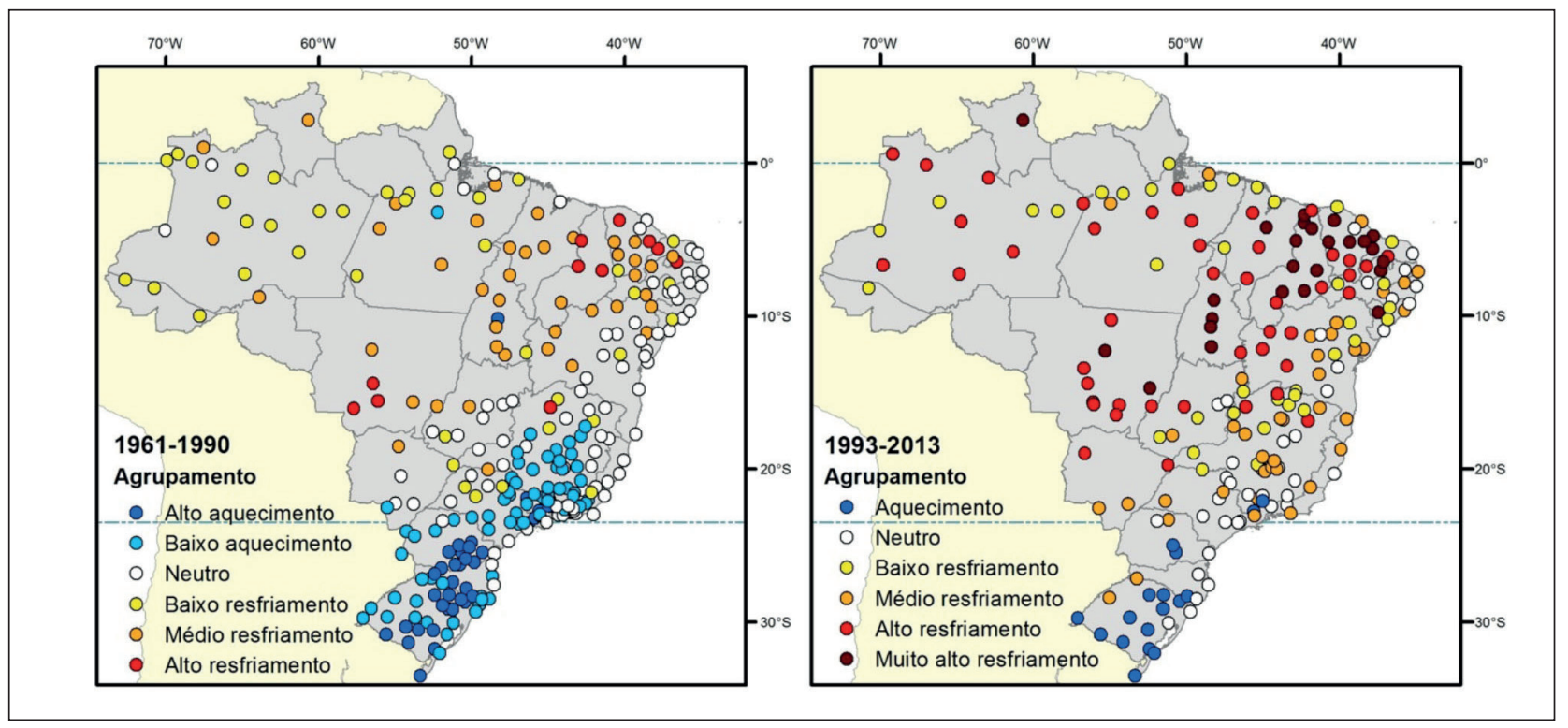

Figura 4 - Mapa do zoneamento das cidades brasileiras por demanda de aquecimento ou resfriamento.

Fonte: Elaboração própria.

\section{Considerações finais}

Para o desenvolvimento de uma proposta de zoneamento climático baseado na demanda de condicionamento térmico, foram utilizados os dados mensais de temperatura média do ar, temperatura média das máximas, temperatura média das mínimas e precipitação, oriundos das Normais Climatológicas (INMET, 2009), por se tratar da maior base de dados climáticos disponível para o Brasil atualmente, e médias mensais do Banco de Dados Meteorológicos para Ensino e Pesquisa do INMET, por conterem dados mais atuais.

A partir destes dados, convertidos em dados horários, foram calculados os números de horas de graus-dia de aquecimento (GDA) e de graus-dia de resfriamento (GDR). Adotou-se, devido à diversidade climática existente no Brasil, a temperatura de neutralidade definida de acordo com o modelo adaptativo de De Dear e Brager (2002), para se estabelecer as temperaturas de base diferenciadas para cada localidade para aquecimento e resfriamento, de forma a considerar a aclimatação das populações ao clima. Adotou-se a faixa de aceitabilidade de $\mathrm{Tn}+2,5^{\circ} \mathrm{C}$ para o cálculo do GDR e de Tn- $5^{\circ} \mathrm{C}$ para o cálculo do GDA. A opção pelo limite de $\mathrm{Tn}-5^{\circ} \mathrm{C}$ se deu devido a esse limite não gerar demanda de aquecimento em locais de clima quente.
Além disso, esse limite resulta em temperaturas de base próximas à $18^{\circ} \mathrm{C}$ em cidades de climas amenos, em conformidade ao valor usualmente adotado em países de clima temperado.

Após a realização do cálculo de GDR e GDA para o universo de 317 cidades, no caso da base de dados das Normais Climatológicas, e de 204 cidades, no caso da base de dados do INMET. As cidades foram agrupadas por similaridade de demanda de aquecimento e resfriamento por meio da técnica de cluster. Para GDR, testou-se agrupamentos com 3, 5 e 7 grupos, o que permitiu constatar que o agrupamento com melhor representatividade e significância estatística correspondeu ao agrupamento com cinco grupos para ambas bases de dados, sendo este então o adotado. De modo geral, observa-se um aumento de GDR e redução de GDA entre o período de 1961-1990, essa tendência pode ser parcialmente explicada pelo fato de que há menos dados provenientes de estações nas regiões de clima mais ameno, Sul e Sudeste, no ciclo mais atual. Além disso, a avaliação das cidades que possuem dados em ambos os períodos permite observar o aumento destacado do número de GDR em todo o país.

Em ambas as bases de dados, seis agrupamentos finais foram obtidos pela comparação da ocorrência das cidades nos respectivos grupos de GDR e GDA. Para os dados das Normais Climatológicas 
(1961-1990) obteve-se então um agrupamento final de seis grupos, quais são: demanda de aquecimento baixa; demanda de aquecimento alta; demanda de resfriamento baixa; demanda de resfriamento moderada; demanda de resfriamento elevada; sem demanda de aquecimento e resfriamento. Já para os dados do Banco de Dados do INMET (1993-2013), o agrupamento final é: demanda de aquecimento; demanda de resfriamento baixa; demanda de resfriamento média; demanda de resfriamento alta; demanda de resfriamento muito alta; sem demanda de aquecimento e resfriamento. Esses grupos gerados apresentaram-se coerentes, significantes e com baixo desvio padrão considerando a extensão e diversidade do território brasileiro.

Como próximas etapas de trabalho prevê-se a avaliação da influência de outras variáveis (como altitude, ventos e relevo) no zoneamento e expansão da base de dados utilizada para refinamento do zoneamento climático baseado na demanda de condicionamento térmico de edificações residenciais.

\section{Referências}

Associação Sul Brasileira de Refrigeração, Ar Condicionado, Aquecimento e Ventilação - ASBRAV. (2014). Setor de Refrigeração, Ar Condicionado, Aquecimento e Ventilação projeta crescimento de 10\% no país. Recuperado em 14 de novembro, 2016 de: http://asbrav.org.br/setor-de-refrigeracao-ar-condicionado-aquecimento-e-ventilacao-projeta-crescimento-de-10-no-pais/

Assis, E. S. (2001). Método Integrado de Análise Climática para Arquitetura Aplicado à Cidade de Belo Horizonte, MG. In Encontro Nacional e Encontro Latino-Americano Sobre Conforto no Ambiente Construído. São Pedro: ANTAC.

Auliciems, A. (1981). Psycho-Physiological Criteria for Global Thermal Zones of Building Design. International Journal of Biometeorology.

Borah, P., Singh, M. K. \& Mahapatra, S. (2015). Estimation of degree-days for different climatic zones of North-East India. Sustainable Cities and Society, 14, 70-81.

Bussab, W. O., Miazaki, E. S. \& Andrade, D. F. (1990). Introdução à Análise de Agrupamentos. São Paulo: Associação Brasileira de Estatística.
Cartalis, C., Synodinou, A., Proedrou, M., TsangrassouliS, A. \& Santamouris, M. (2001). Modifications in energy demand in urban areas as a result of climate changes: an assessment for southeast Mediterranean region. Energy Conversion and Management, 42, 1647-1656.

Christenson M.; Manz H.; \& Gyalistras D. (2006). Climate Warming Impact on Degreedays and Building Energy Demand in Switzerland. Energy Convers Manage, 47, 671-686

Day, T. (2006). Degree-days: theory and application TM4. London: The Chartered Institution of Building Services Engineers (CISBE).

De Dear, R. J., \& Brager, G. S. (2002). Thermal Comfort in Naturally Ventilated Buildings: revisions to ASHRAE Standard 55. Energy and Buildings, 34, 549-561.

Departamento Nacional de Meteorologia - DNMET. (1992). Normais climatológicas 1961-1990. Brasília: DNMET.

Departament Of Energy - DOE. (2010). High-Performance Home Technologies: Guide to Determining Climate Regions by County. Washington: DOE.

Empresa de Pesquisa Energética - EPE. (2016). Balanço Energético Nacional 2010: Ano base 2015. Rio de Janeiro: EPE.

Farias, A. A., Cesar, C. C. \& Soares, J. F. (2003). Introdução à estatística. Rio de Janeiro: Livros técnicos e científicos.

Humphreys, M. (1978). Outdoor Temperatures and Comfort Indoors. Building Research \& Information, 6, 92.

Instituto Nacional de Meteorologia - INMET. (2009). Normais climatológicas do Brasil: 1961 - 1990: Edição revisada e ampliada. Brasília: INMET.

Instituto Nacional de Meteorologia - INMET. (2016). Banco de Dados Meteorológicos para Ensino e Pesquisa - BDMEP. Recuperado em 14 de novembro de 2016, de: http://www.inmet.gov.br/projetos/rede/pesquisa/

Kottek, M., Grieser, J.; Beck, C.; Rudolf, B.; \& Rubel, F. (2006). World Map of the Köppen-Geiger Climate Classification Updated. Meteorologische Zeitschrift, 15, 259-263.

Lam J. C., Wan K. K. W., Pan W. \& Li D. H. W. (2011). Long-term Trends of Degree-Days In the 20th and 21st Centuries and Heating and Cooling Energy Use Implications: Research report. Hong Kong: Department of Civil and Architectural Engineering. 
Lee K., \& Levermore G. J. (2010). Weather Data for Future Climate Change for South Korean Building Design: Analysis for Trends. Architectural Science Review, 53, 157-171.

Li, D.H.W., Yang, L. \& Lam, J.C. (2012). Impact of climate change on energy use in the built environment in different climate zones - A review. Energy, 42, 103-112.

Nicol, J. F., \& Humphreys, M. A. (2002). Adaptive Thermal Comfort and Sustainable Thermal Standards for Buildings. Energy and Buildings, 34, 563-572.

Peel, M. C., Finlayson, B. L., \& McMahon, T. A. (2007). Updated World Map of the Köppen-Geiger Climate Classification. Hydrology and Earth System Sciences, 11, 1633-1644.

Pereira, I. M., \& Assis, E. S. (2010). Avaliação de Modelos de Índices Adaptativos para Uso no Projeto Arquitetônico Bioclimático. Ambiente Construído, 10, 37-49.

Pilli-Sihvola K.; Aatola P.; Ollikainen M.; \& Tuomenvirta H. (2010). Climate and Electricity Consumption: Witnessing Increasing or Decreasing Use and Costs? Energy Policy, 38, 2409-2419.
ProgramaNacionaldeEficiênciaEnergéticaem Edificações - PROCEL (2007). Pesquisa de Posse de Equipamentos e Hábitos de Consumo de Energia. Eletrobrás. Recuperado em 14 de novembro de 2016, de: http://www.procel.gov. br/data/Pages/LUMIS05070313PTBRIE.htm

Reddy, T. A. (2011). Applied Data Analysis and Modeling for Energy Engineers and Scientists. London: Springer.

Roshan, G.R., Ghanghermeh, A. A. \& Attia, S. (2017). Determining new threshold temperatures for cooling and heating degree day index of different climatic zones of Iran. Renewable Energy, 101, 156-167.

Recebido: Nov. 15, 2016

Aprovado: Jan. 16, 2017

urbe. Revista Brasileira de Gestão Urbana (Brazilian Journal of Urban Management), 2017, 9(Supl. 1), 286-300 